\title{
Real-Time Color Image Watermarking Based on D-SVD Scheme
}

\author{
Cheng-Fa Tsai and Wen-Yi Yang \\ Department of Management Information Systems, \\ National Pingtung University of Science and Technology, \\ 91201 Pingtung, Taiwan \\ cftsai@mail.npust.edu.tw
}

\begin{abstract}
This investigation presents a robust digital watermarking scheme for copyright protection, called D-SVD. The proposed scheme integrates discrete cosine transform (DCT) with singular value decomposition (SVD). In contrast to traditional methods based on DCT watermarking schemes, in which watermark messages are embedded directly on DCT frequency coefficients, the proposed approach emphasizes that watermark message bit is embedded on the blocks of the DCT coefficient's singular value within an original color image. Experimental results demonstrate that the quality of watermarked image is robust under compression, noises, filtering and various attacks. In addition, it is observed that the proposed D-SVD algorithm can obtain larger NC and PSNR values than some existing well-known methods, and can successfully resist attacks such as cropping, blurring, reshaping, adding noise and JPEG compression.
\end{abstract}

Keywords: digital watermarking, copyright protection, image processing.

\section{Introduction}

Digital watermarking algorithms are generally developed to verify authorship or to protect copyright [1], [4]-[18]. Numerous watermarking schemes that embed the watermark into transformed frequency coefficients have been presented recently, due to the robustness consideration such as DCT [1], [3], [13], [16] and wavelet [2], [18]. This methodology is unlike conventional approaches based on the transformed coefficient. This study proposes a new method that embeds the watermark into the singular value of the DCT coefficient blocks within an original color image, and does not require the original image when performing extraction of watermark procedure. The proposed scheme first calculates the frequency coefficient of luminance within the original color image using DCT, and decomposes the coefficient to computes its singular value after. The watermarks are then embedded on the singular value. Experimental results indicate that the presented technique produces a high-quality watermarked image and a robust embedding watermark. 


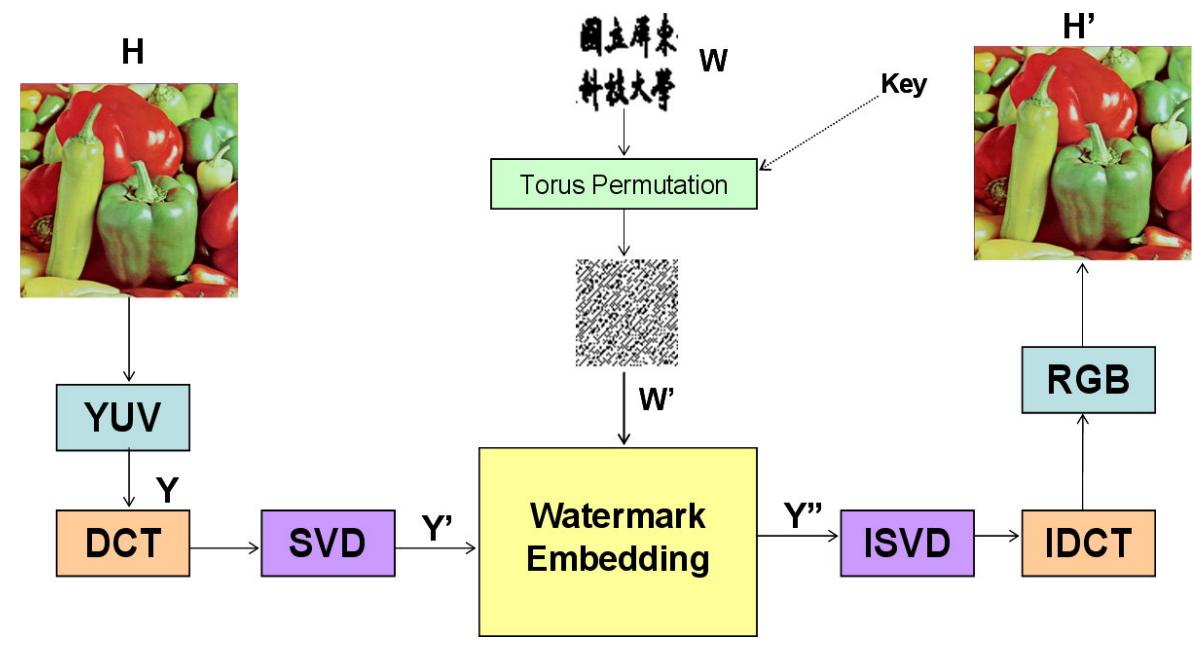

Fig. 1. The proposed watermark embedding process

\section{Embedding Process}

According to illustrated in Fig. 1, the proposed process embeds an $n \times n$ watermark image $W$ into an $N \times N$ size original image $H$, with secret keys (key) for security. The steps of watermark embedding process are provided as follows:

Step 1: Transform the RGB color space of the original image $H$ to the YUV color space. The $Y$ (luminance) component has selected from YUV to apply the DCT frequency transformation, since the luminance has the same range as the gray image between 0 and 255. The original image luminance component $Y$ is split into non-overlapping $8 \times 8$ or $4 \times 4$ pixel blocks $B_{k}$, where $k=1,2,3, \ldots, M$, and $M$ represents the numbers of blocks. Each block $B_{k}^{\prime}$ is then individually transformed to a frequency coefficient using the two-dimension DCT.

Step 2: Compute the singular value of each frequency coefficient block $B_{k}^{\prime}$ by singular value decomposition.

Step 3: Compute $N_{s}=\|s\|+1$ and $D=\left\lfloor N_{s} / d\right\rfloor$, where $s=\left(\lambda_{1}^{k}, \lambda_{2}^{k}, \ldots, \lambda_{N}^{k}\right)$ denotes a vector formed to the singular values of each block $B_{k}^{\prime}$, and $d_{k}$ represents the quantization level for correlating $N_{s}$ with $B_{k}^{\prime}$.

Step 4: Shuffle the watermark image for embedding, to prevent tampering with the watermark form. The proposed approach employs Torus automorphism, which is an image permutation mapping function to break up a watermark image randomly. The number of Torus permutation transformations on the watermark image must be maintained as a secret key for the extracting process.

Step 5: Embed each bit bi (0 or 1$)$ of watermark image by the adjustment integer number $D$. If $b i=0$, then $D$ is odd, while if $b i=1$, then $D$ is even. 
Step 6: Compute $N_{s}^{\prime}=d_{k} \times D+d_{k} / 2$ and the modified singular value $\left(\delta_{1}^{k}, \delta_{2}^{k}, \ldots\right.$, $\left.\delta_{N}^{k}\right)$, where $\left(\delta_{1}^{k}, \delta_{2}^{k}, \ldots, \delta_{N}^{k}\right)=\left(\lambda_{1}^{k}, \lambda_{2}^{k}, \ldots, \lambda_{N}^{k}\right) \times\left(N_{s}^{\prime} / N_{s}\right)$.

Step 7: Compute the watermarked block $B_{k}^{\prime \prime}$ with the modified singular value $\left(\lambda_{1}^{k}, \lambda_{2}^{k}, \ldots, \lambda_{N}^{k}\right)$.

Step 8: Inversely transform each $B_{k}^{\prime \prime}$ to luminance image domain using IDCT.

Step 9: Reconfigure the watermarked image from all blocks to obtain the RGB color space.

\section{Extracting Process}

Fig. 2 displays the extracting process utilized in the proposed scheme. The steps of extracting the watermark from the watermarked image are given as follows:

Step 1: Transform the watermarked image $Y_{w}$ to the YUV color space, and then place the luminance component $Y$ into non-overlapping $8 \times 8$ or $4 \times 4$ pixel blocks $\tilde{B}_{k}$.

Step 2: Transform each block $\tilde{B}_{k}$ to the frequency coefficient by two-dimension DCT, and then compute the singular value of each frequency coefficient block $\tilde{B}_{k}^{\prime}$ using the singular value decomposition.

Step 3: Compute $\tilde{N}_{s}=\|\tilde{s}\|+1 ; \tilde{D}=\left\lfloor\tilde{N}_{s} / d_{k}\right\rfloor$, where $\tilde{s}=\left(\delta_{1}^{k}, \delta_{2}^{k}, \ldots, \delta_{N}^{k}\right)$ represents a vector formed to the singular values of each block $\tilde{B}_{k}^{\prime}$.

Step 4: If $\tilde{D}$ is odd, then the embedded watermark bit is 0 . Otherwise, if $\tilde{D}$ is even, then the embedded bit is 1 .

Step 5: Reshape the randomly watermark $W^{\prime}$, by performing the Torus permutation transformation once to reconfigure the recovery watermark using the appropriate secret key value.

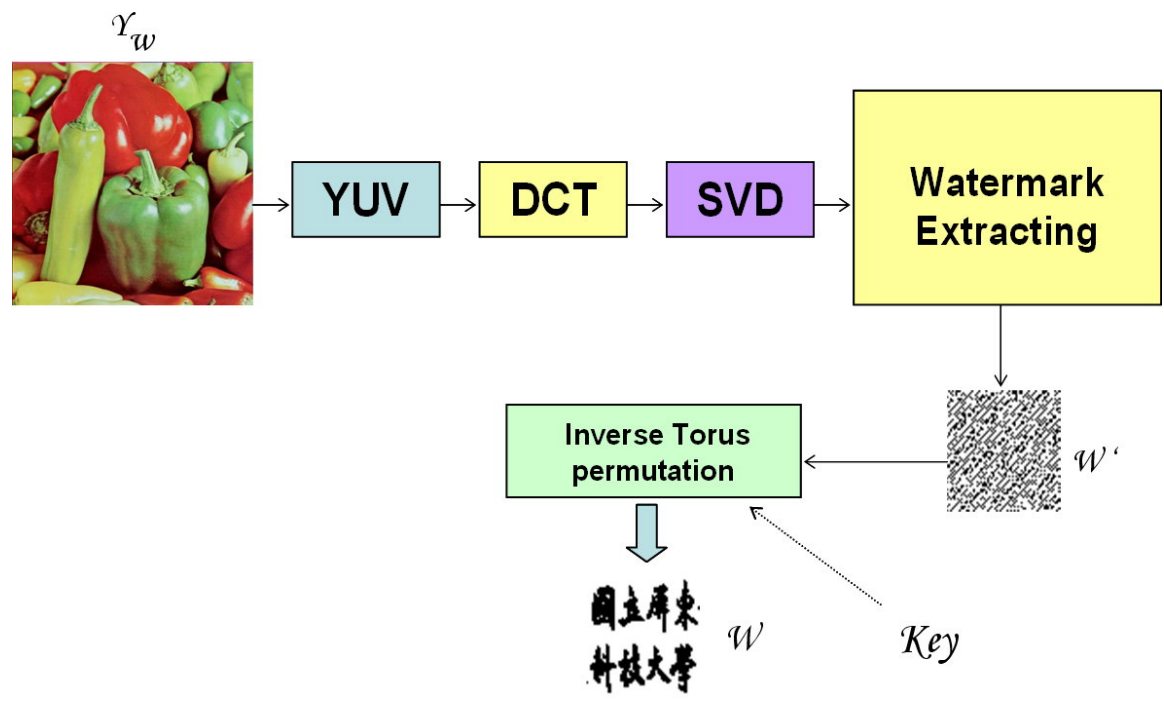

Fig. 2. The proposed watermark extracting process 


\begin{tabular}{|c|c|c|c|c|c|c|}
\hline \multirow{2}{*}{\multicolumn{2}{|c|}{$\begin{array}{c}\text { Host Image: lena } \\
\text { JPEG Quality }\end{array}$}} & \multicolumn{5}{|c|}{ Attacked by JPEG compression } \\
\hline & & \multirow{2}{*}{$\begin{array}{c}100 \\
40.881\end{array}$} & \multirow{2}{*}{$\frac{90}{40.394}$} & \multirow{2}{*}{$\frac{80}{39.763}$} & \multirow{2}{*}{$\frac{70}{39.330}$} & \multirow{2}{*}{$\frac{60}{36.453}$} \\
\hline & PSNR & & & & & \\
\hline & $\mathrm{NC}$ & 1.000 & 0.9976 & 0.9980 & 0.9970 & 0.9897 \\
\hline \multirow{2}{*}{$\begin{array}{l}\text { Wang's } \\
\text { method [3] }\end{array}$} & PSNR & 38.633 & 38.366 & 38.007 & 37.679 & 35.532 \\
\hline & NC & 0.9941 & 0.9921 & 0.9625 & 0.9249 & 0.7768 \\
\hline \multirow{2}{*}{$\begin{array}{l}\text { Liu's } \\
\text { method [15] }\end{array}$} & PSNR & 36.837 & 36.347 & 38.018 & 39.993 & 36.692 \\
\hline & NC & 0.9965 & 0.9981 & 0.9795 & 0.9204 & 0.9179 \\
\hline \multicolumn{2}{|c|}{ JPEG Quality } & 50 & 40 & 30 & 20 & 10 \\
\hline \multirow{2}{*}{ D-SVD } & PSNR & 33.826 & 33.533 & 32.683 & 31.163 & 28.177 \\
\hline & $\mathrm{NC}$ & 0.9658 & 0.7964 & 0.6714 & 0.2759 & -0.217 \\
\hline \multirow{2}{*}{$\begin{array}{l}\text { Wang's } \\
\text { method [3] }\end{array}$} & PSNR & 33.385 & 33.057 & 32.318 & 30.839 & 28.093 \\
\hline & NC & 0.7027 & 0.5773 & 0.4064 & 0.3096 & 0.2462 \\
\hline \multirow{2}{*}{$\begin{array}{l}\text { Liu's } \\
\text { method [15] }\end{array}$} & PSNR & 34.082 & 33.661 & 32.922 & $\mathbf{3 1 . 3 1 7}$ & 28.362 \\
\hline & $\mathrm{NC}$ & 0.9263 & 0.8633 & 0.8223 & 0.5986 & 0.2446 \\
\hline
\end{tabular}

Fig. 3. Comparison of the proposed D-SVD algorithm and Wang's and Liu's methods with extracted watermark $\mathrm{NC}$ value and the PSNR of watermarked through attacked by JPEG with different compression qualities (using Lena as testing image)

\begin{tabular}{|c|c|c|c|c|}
\hline \multicolumn{2}{|c|}{ Host Image: lena } & \multicolumn{3}{c|}{ Attacked by blurring } \\
\hline \multicolumn{2}{|c|}{ blurring times } & 1 & 2 & 3 \\
\hline \multirow{2}{*}{ D-SVD } & PSNR & 38.242 & 34.391 & 31.285 \\
\cline { 2 - 5 } & NC & $\mathbf{0 . 9 5 1 2}$ & $\mathbf{0 . 8 2 4 7}$ & $\mathbf{0 . 6 9 7 3}$ \\
\hline \multirow{2}{*}{ Wang's method [3] } & PSNR & 37.658 & 34.185 & 31.192 \\
\cline { 2 - 5 } & NC & $\mathbf{0 . 5 4 3 7}$ & $\mathbf{0 . 3 4 2 2}$ & $\mathbf{0 . 2 6 5 2}$ \\
\hline
\end{tabular}

Fig. 4. Comparison of the proposed D-SVD algorithm and Wang's method with extracted watermark NC value and the PSNR of watermarked through attack by blurring (using Lena as testing image) 


\begin{tabular}{|c|c|c|c|}
\hline Host Image: lena & \multicolumn{3}{|c|}{ Attacked by blurring } \\
\hline Add blurring times & 1 & 2 & 3 \\
\hline D-SVD & 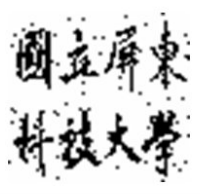 & $\begin{array}{l}4{ }^{4} \\
4 \\
4\end{array}$ & 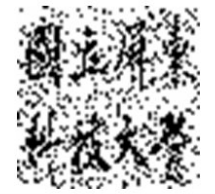 \\
\hline Wang's method [3] & 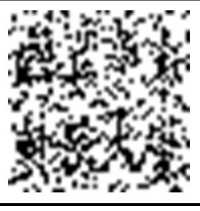 & 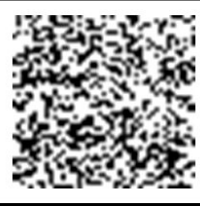 & 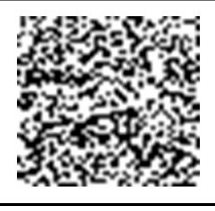 \\
\hline
\end{tabular}

Fig. 5. The outcome of watermarks after attack by blurring with three times using the proposed D-SVD and Wang's method [3]

\begin{tabular}{|l|c|c|c|c|c|c|c|}
\hline \multicolumn{6}{|c|}{ Host Image: lena } & \multicolumn{7}{c|}{ Attacked by noise (salt \& pepper) } \\
\hline \multicolumn{2}{|c|}{ Add Noise times } & 1 & 2 & 3 & 4 & 5 & 6 \\
\hline \multirow{2}{*}{ D-SVD } & PSNR & 34.293 & 31.604 & 30.152 & 28.844 & 27.912 & 27.258 \\
\cline { 2 - 8 } & NC & 0.960 & 0.9106 & 0.8774 & 0.8247 & 0.7769 & 0.7520 \\
\hline \multirow{2}{*}{$\begin{array}{l}\text { Wang's } \\
\text { method [3] }\end{array}$} & PSNR & 33.854 & 31.258 & 29.799 & 28.709 & 27.808 & 27.203 \\
\cline { 2 - 8 }$y$ & NC & 0.8361 & 0.7511 & 0.6681 & 0.6246 & 0.5812 & 0.5091 \\
\hline
\end{tabular}

Fig. 6. Comparison of the proposed D-SVD algorithm and Wang's method [3] with extracted watermark NC value and the PSNR of watermarked through attack by adding noise (using Lena as testing image and using Salt \& Pepper as noise)

\begin{tabular}{|c|c|c|c|c|c|c|}
\hline Host Image: lena & \multicolumn{6}{|c|}{ Attacked by noise (salt \& pepper) } \\
\hline Add Noise times & 1 & 2 & 3 & 4 & 5 & 6 \\
\hline D-SVD & 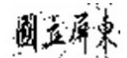 & 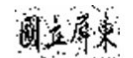 & 4,4 & 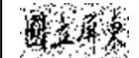 & W\% & a \\
\hline watermark(recovered) & 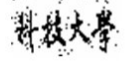 & $\mathrm{C}_{\mathrm{d}} \mathrm{x}$ & As & Nox & , & $4 x$ \\
\hline $\begin{array}{l}\text { Wang's method [3] } \\
\text { watermark(recovered) }\end{array}$ & 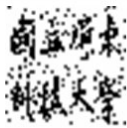 & 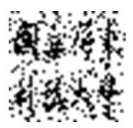 & 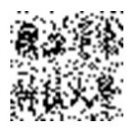 & 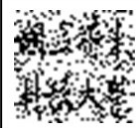 & 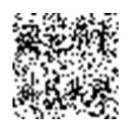 & 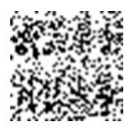 \\
\hline
\end{tabular}

Fig. 7. The outcome of watermarks after attack by adding noise with six times using the proposed D-SVD and Wang's method [3] 


\section{Simulation Result}

An experiment was performed using an original image of size $512 \times 512$ with true color, and a watermark image of size $64 \times 64$ with binary image. The experiment was performed on a personal computer with an Intel Pentium 4, 3-GHz and 512 M RAM. To verify the performance of the proposed D-SVD algorithm, some experiments were conducted to compare the proposed D-SVD algorithm and Wang's and Liu's methods [3], [15]. Fig. 3-7 show some experiment results. Fig. 3 presents the comparison of the proposed D-SVD algorithm and Wang's and Liu's methods with extracted watermark NC (Normalized Correlation) value and the PSNR (Peak Signal to Noise Ratio) of watermarked through attacked by JPEG with different compression qualities (using Lena as testing image). The human eye can generally judge the quality of a processed image, but not very objectively. Some objective measures are available to verify the quality of a compressed image. For instance mean square error (MSE), signal/noise relation (SNR) and Peak Signal-to-Noise Ratio (PSNR) are generally used, and are formulated as follows.

$$
\begin{gathered}
M S E=\frac{1}{M \times N} \sum_{x=0}^{M-1} \sum_{y=0}^{N-1}[\hat{I}(x, y)-I(x, y)]^{2} \\
S N R=10 \log _{10} \frac{\frac{1}{M \times N} \sum_{x=0}^{M-1} \sum_{y=0}^{N-1}\left[\hat{I}(x, y)^{2}\right]}{M S E} \\
P S N R=10 \log _{10} \frac{255^{2}}{M S E}
\end{gathered}
$$
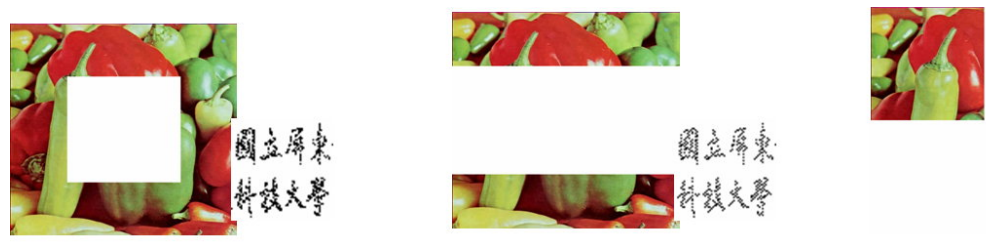

Fig. 8. The outcome of watermarks after attack by cropping with different percentages $(25 \%, 50 \%$, and $75 \%$ ) using the proposed D-SVD approach (cropping 25\%, $\mathrm{PSNR}=11.246 \mathrm{~dB}, \mathrm{NC}=0.8999$; cropping $50 \%, \mathrm{PSNR}=7.5894 \mathrm{~dB}, \mathrm{NC}=0.7969$; cropping $75 \%, \mathrm{PSNR}=5.692 \mathrm{~dB}, \mathrm{NC}=0.6919)$

Additionally, Normalized Correlation (NC) is used to evaluate the similarity degree between original watermark and extracting watermark. Fig. 4 lists the comparison of the proposed D-SVD algorithm and Wang's method with extracted watermark NC value and the PSNR of watermarked through attack by 
blurring (using Lena as testing image). Fig. 5 illustrates the outcome of watermarks after attack by blurring with three times using the proposed D-SVD and Wang's method [3]. Fig. 6 displays the result after an attack by adding noise. Fig. 8 depicts the outcome of watermarks after attack by different percentages cropping $(25 \%, 50 \%$, and $75 \%$ ) using the proposed D-SVD approach (notably, cropping $25 \%, \mathrm{PSNR}=11.246 \mathrm{~dB}, \mathrm{NC}=0.8999$; cropping $50 \%, \mathrm{PSNR}=7.5894 \mathrm{~dB}$, $\mathrm{NC}=0.7969$; cropping $75 \%, \mathrm{PSNR}=5.692 \mathrm{~dB}, \mathrm{NC}=0.6919)$. It is found that the outcome of watermarks after attack by high percentage cropping using D-SVD approach is still clear. However, it is difficult to find watermark after attack by high percentage cropping using Wang's method.

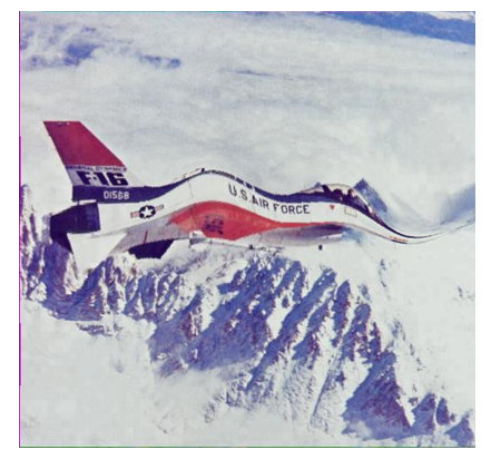

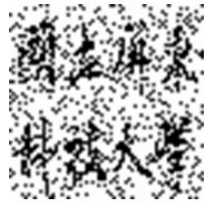

(b)

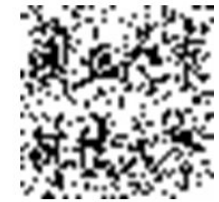

(c)

(a)

Fig. 9. The outcome of watermarks after attack by reshaping using the proposed DSVD approach and Wang's method [3] (a)using F16 as reshaping testing image (b) using D-SVD algorithm (c) using Wangs method

Fig. 9 shows the result of watermarks after attack by reshaping using the proposed D-SVD approach $(\mathrm{PSNR}=20.4762, \mathrm{NC}=0.6895)$ and Wang's method [3] $(\mathrm{PSNR}=20.3242, \mathrm{NC}=0.66040)$, and $\mathrm{F} 16$ airplane was used as reshaping testing image. From Fig. 3-9, it is observed that the proposed D-SVD algorithm can obtain larger NC and PSNR values than Wang's and Liu's methods, and can successfully resist attacks such as cropping, blurring, reshaping, adding noise and JPEG compression. Hence, the proposed image watermarking technique is robust and imperceptible.

\section{Conclusion}

This investigation presents a new scheme for embedding digital watermark into a color image. The embedding and extracting processes are based on D-SVD. Experimental results reveal that the proposed method can successfully resist attacks such as cropping, blurring, reshaping, adding noise and JPEG compression. 
Therefore, the proposed image watermarking technique is robust and imperceptible. Additionally, the proposed approach can retrieve the embedded information without accessing the original image. Experimental results demonstrate that the proposed schemes outperform Wang's and Liu's schemes.

Acknowledgments. The author would like to thank the National Science Council of Republic of China for financially supporting this research under contract no. NSC 94-2213-E-020-002.

\section{References}

1. Narges, A., Reza, S.: A Novel DCT-based Approach for Secure Color Image Watermarking. In: IEEE Computer Society Proceedings of the International Conference on Information Technology: Coding and Computing (2004)

2. Paul, B., Ma, X.-H.: Image Adaptive Watermarking Using Wavelet Domain Singular Value Decomposition. IEEE Transactions on Circuits and Systems for video Technology 15, 96-102 (2005)

3. Wang, Y., Pearmain, A.: Blind image data based on self reference. Pattern Recognition Letters, 1681-1689 (2004)

4. Chen, L.-H., Lin, J.-J.: Mean quantization based image watermarking. Image and Vision Computing, 717-727 (2003)

5. Chung, K.-L., Shen, C.-H., Chang, L.-C.: A novel SVD and VQ-based image hiding schemes. Pattern Recognition Letters, 1051-1058 (2001)

6. Chu, W.-C.: DCT-Based Image Watermarking Using Sub-sampling. IEEE Transactions on Multimedia 5, 34-38 (2003)

7. Voyatzis, G., Pitas, I.: Chaotic Mixing of Digital Images and Applications to watermarking. In: Proceedings of European Conference on Multimedia Applications Service and Techniques, vol. 2, Belgium, European (1996)

8. Hsu, C.-T., Wu, J.-L.: Hidden Digital Watermarks in Images. IEEE Transactions on Image Processing 8 (1999)

9. Huang, F., Guan, Z.-H.: A hybrid SVD-DCT watermarking method based on LPSNR. Pattern Recognition Letters on 25, 1769-1775 (2004)

10. Hwang, M.-S., Chang, C.-C., Hwang, K.-F.: A Watermarking Technique Based on one-way Hash function. IEEE Transactions on Consumer Electronics 45, 286-294 (1999)

11. Huang, J., Shi, Y.-Q., Shi, Y.: Embedding Image Watermarks in DC Components. IEEE Transactions on Circuits and Systems for Video Technology 10, 974-979 (2000)

12. Cox, I.-J., Kilian, J., Leighton, F.-T., Shamoon, T.: Secure spread spectrum watermarking for multimedia. IEEE Transactions on Image Processing 6, 1673-1687 (1997)

13. Lin, S.-F., Chen, C.-F.: A Robust DCT-BASED Watermarking for copyright protection. IEEE Transactions on Consumer Electronics 46, 415-421 (2000)

14. Li, C.-T.: Digital fragile watermarking scheme for authentication of JPEG images. IEEE Image Signal Processing 151, 460-466 (2004)

15. Liu, R., Tan, T.: An SVD-Based Watermarking Scheme for Protecting Right Ownership. IEEE Transactions on Multimedia 4, 121-128 (2002) 
16. Li, X.-Q., Xue, X.-Y.: Improved Robust Watermarking in DCT Domain for Color Images. In: IEEE Computer Society Proceedings of the 18th International Conference on Advanced information Networking and Application (2004)

17. Shih, F.-Y., Wu, Y.-T.: Enhancement of image watermark retrieval based on genetic algorithms. Journal of Visual Communication and Image Representation, 115-133 (2005)

18. Tsai, M.-J., Yu, K.-Y., Chen, Y.-Z.: Joint Wavelet and Spatial Transformation for Digital Watermarking. IEEE Transactions on Consumer Electronics 46, 241-245 (2000) 